\title{
A discussion concerning the existence results for the Sobolev-type Hilfer fractional delay integro-differential systems
}

\author{
K. Kavitha ${ }^{1}$, Kottakkaran Sooppy Nisar ${ }^{2 *}$, Anurag Shukla ${ }^{3}$, Velusamy Vijayakumar ${ }^{1}$ and \\ Shahram Rezapour ${ }^{4,5^{*}}$ iD
}

${ }^{*}$ Correspondence:

n.sooppy@psau.edu.sa;

sh.rezapour@azaruniv.ac.ir;

sh.rezapour@mail.cmuh.org.tw;

rezapourshahram@yahoo.ca

${ }^{2}$ Department of Mathematics

College of Arts and Sciences, Prince

Sattam bin Abdulaziz University,

Wadi Aldawaser 11991, Saudi Arabia

${ }^{4}$ Department of Mathematics,

Azarbaijan Shahid Madani

University, Tabriz, Iran

Full list of author information is

available at the end of the article

\begin{abstract}
The goal of this study is to propose the existence results for the Sobolev-type Hilfer fractional integro-differential systems with infinite delay. We intend to implement the outcomes and realities of fractional theory to obtain the main results by Monch's fixed point technique. Moreover, we show the existence and controllability of the thought about the fractional system with the nonlocal condition. In addition, an application to illustrate the outcomes is also included.
\end{abstract}

MSC: Primary 34K30; 34K40; secondary 47H08; 47H10

Keywords: Generalized Caputo fractional derivative; Hilfer fractional differential equation; Measures of noncompactness; Sobolev-type equations

\section{Introduction}

In recent years, mathematical modeling has been upheld by fractional calculus, with a few outcomes, and fractional operators were demonstrated to be a fantastic instrument to depict the hereditary characteristics of different patterns. As of late, this blend has acquired a lot of significance, basically because fractional differential equations have become amazing assets for displaying a few complex wonders in various assorted and boundless fields of science and engineering; readers are referred to [1-20] and articles [21-37]. Hilfer [38] initiated another kind of derivative, along with Riemann-Liouville and Caputo fractional derivative. Motivated by the monograph, nowadays, several authors focus on these Hilfer fractional differential equations, and we refer to [24, 39-48]. Singh et al. [49] discussed the existence and Ulam stability of solutions for a class of boundary value problems for Hilfertype nonlinear implicit fractional differential equations with instantaneous impulses in Banach spaces.

The differential system with Sobolev-type is frequently evident in the mathematical structure of several physical events similar to the flowing of fluids through fractured rocks, thermodynamics. The readers may refer to [50-56]. Many authors discussed the relations between the asymptotic stability of the zero solution for retarded differential equations

(c) The Author(s) 2021. This article is licensed under a Creative Commons Attribution 4.0 International License, which permits use, sharing, adaptation, distribution and reproduction in any medium or format, as long as you give appropriate credit to the original author(s) and the source, provide a link to the Creative Commons licence, and indicate if changes were made. The images or other third party material in this article are included in the article's Creative Commons licence, unless indicated otherwise in a credit line to the material. If material is not included in the article's Creative Commons licence and your intended use is not permitted by statutory regulation or exceeds the permitted use, you will need to obtain permission directly from the copyright holder. To view a copy of this licence, visit http://creativecommons.org/licenses/by/4.0/. 
and real parts of all characteristic roots of characteristic equations. In [57] the author investigated the asymptotic stability of the zero solution for Caputo-Hadamard fractional dynamic equations on a time scale. These equations guarantee the effectiveness of the zero solution, and several authors reported interesting fixed point results in the framework of complete b-metric spaces, recently, Lazreg et al. [58] established some impulsive Caputo-Fabrizio fractional differential equations in b-metric spaces.

Control hypothesis is a significant region of usage arranged in mathematics which deals with the design and assessment of control structures. The development of modern mathematical control theory is heavily influenced by controllability. The problem of controllability of dynamical systems is commonly employed in control system analysis and design. Fractional-order control systems defined by fractional-order differential equations have gotten a lot of interest in recent years, a wide list of these distributions can be found in $[25,26,28,29,40,43,48,51,56,59-62]$. The controllability of impulsive fractional evolution inclusions with state-dependent delay is demonstrated in [63], which employs a fixed point theorem for condensing maps.

From the above literature survey, to our knowledge the existence and exact controllability of the fractional system have not been studied fully. Motivated by this fact, we consider the Sobolev-type Hilfer fractional integro-differential system of the form

$$
\begin{aligned}
& D_{0^{+}}^{\alpha, \beta}[\mathcal{J} z(t)]=A z(t)+f\left(t, z_{t}, \int_{0}^{t} e\left(t, s, z_{s}\right) d s\right), \quad t \in \mathbb{N}=(0, b], \\
& \mathcal{I}_{0^{+}}^{(1-\alpha)(1-\beta)} z(0)=\phi \in \mathcal{B}_{l},
\end{aligned}
$$

and assume that the system with control has the following form:

$$
\begin{aligned}
& D_{0^{+}}^{\alpha, \beta}[\mathcal{J} z(t)]=A z(t)+f\left(t, z_{t}, \int_{0}^{t} e\left(t, s, z_{s}\right) d s\right)+B u(t), \quad t \in \mathbb{N}=(0, b] \\
& \mathcal{I}_{0^{+}}^{(1-\alpha)(1-\beta)} z(0)=\phi \in \mathscr{B}_{l},
\end{aligned}
$$

where $D_{0^{+}}^{\alpha, \beta}$ stands for Hilfer fractional derivative of type $\frac{1}{2}<\beta<1$, order $0 \leq \alpha \leq 1$. The state $z(\cdot)$ takes values in a Banach space along with the norm $\|\cdot\|, A$ is the infinitesimal generator of a $C_{0}$-semigroup. The control function $u(\cdot) \in L^{2}(\mathbb{N}, \mathbb{U})$. The histories $z_{t}:(-\infty, 0] \rightarrow \mathscr{B}_{l}, z_{t}(s)=z(t+s), s \leq 0$ are associated with phase space $\mathscr{B}_{l}$. Additionally, a bounded linear operator $B: \mathbb{U} \rightarrow Z, \mathbb{U} \in Z . f: \mathbb{N} \times \mathscr{B}_{l} \times Z \rightarrow Z$ and $e: \mathbb{N} \times \mathbb{N} \times \mathscr{B}_{l} \rightarrow Z$ are given functions.

We organize the remaining part of our article as follows: Some new notations, important facts, lemmas, vital definitions, and theoretical results are recalled in Sect. 2. Section 3 provides the existence of fractional system (1.1)-(1.2) which is proven by Monch's fixed point theorem. We extended the study to deal with the exact controllability for (1.3)-(1.4) in Sect. 4. In Sect. 5, we discuss the system with nonlocal conditions. Finally, we end with Sect. 6, which presents our conclusions.

\section{Preliminaries}

We review the essential hypothesis which is utilized all through the work in request to acquire new outcomes. Let $v=\alpha+\beta-\alpha \beta$, we have $(1-v)=(1-\alpha)(1-\beta)$. We define 
$\mathcal{C}_{1-v}(\mathbb{N}, Z)=\left\{z: t^{1-v} z(t) \in \mathcal{C}(\mathbb{N}, Z)\right\}$ with $\|\cdot\|_{v}$ defined by $\|z\|_{v}=\sup \left\{t^{1-v}\|z(t)\|, t \in \mathbb{N}\right\}$. Suppose $\mathcal{C}(\mathbb{N}, Z): \mathbb{N} \rightarrow Z$ with $\|Z\|_{\mathcal{C}}:=\sup _{t \in \mathbb{N}}\|z(t)\|$ for $z \in \mathcal{C}$, and we introduce $A: D(A) \subset$ $Z \rightarrow Z, \mathcal{J}: D(A) \subset Z \rightarrow Z$ is satisfied, refer to [53].

$\left(\mathbf{F}_{1}\right)$ The linear operators $A$ and $\mathcal{J}$ are closed.

$\left(\mathbf{F}_{2}\right) D(\mathcal{J}) \subset D(A), \mathcal{J}$ is bijective.

$\left(\mathbf{F}_{3}\right) \mathcal{J}^{-1}: Z \rightarrow D(\mathcal{J})$ is continuous.

In addition, from $\left(\mathbf{F}_{1}\right),\left(\mathbf{F}_{2}\right), \mathcal{J}^{-1}$ is closed. Applying the closed graph theorem and $\left(\mathbf{F}_{3}\right)$, we obtain the boundedness of $A \mathcal{J}^{-1}: Z \rightarrow Z$. Designate $\left\|\mathcal{J}^{-1}\right\|=\widetilde{\mathcal{J}}_{m}$ and $\|\mathcal{J}\|=\mathcal{J}_{m}$.

Definition 2.1 The fractional integral of order $\alpha \in(0,1)$ of $f:[b,+\infty) \rightarrow \mathbb{R}$ is the function $I_{b^{+}}^{\alpha} f$ of the form

$$
I_{b^{+}}^{\alpha} f(t)=\frac{1}{\Gamma(\alpha)} \int_{b}^{t} \frac{f(s)}{(t-s)^{1-\alpha}} d s, \quad t>b ; \alpha>0 .
$$

Definition 2.2 The Riemann-Liouville derivative of order $\alpha \in[m-1, m), m \in \mathbb{Z}^{+}$for $f:[b,+\infty) \rightarrow \mathbb{R}$, the function ${ }^{L} D^{\alpha}{ }^{+} f$ of the form

$$
{ }^{L} D^{\alpha} b^{+} f(t)=\frac{1}{\Gamma(m-\alpha)} \frac{d^{m}}{d t^{m}} \int_{b}^{t} \frac{f(s)}{(t-s)^{\alpha+1-m}} d s, \quad t>b, m-1 \leq \alpha<m .
$$

Definition 2.3 The Hilfer fractional derivative of order $0 \leq \alpha \leq 1$ and type $0<\beta<1$ for $f(t)$ of the form

$$
D_{b^{+}}^{\alpha, \beta} f(t)=\left(I_{b^{+}}^{\alpha(1-\beta)} D\left(\mathcal{I}_{b^{+}}^{(1-\alpha)(1-\beta)} f\right)\right)(t)
$$

\section{Remark 2.4}

(i) In case $\alpha=0, b=0$, the Hilfer fractional differential is identical to the classical Riemann-Liouville fractional derivative for $f$ of the form

$$
D_{0^{+}}^{0, \beta} f(t)=\frac{d}{d t} \mathcal{I}_{0^{+}}^{1-\beta} f(t)={ }^{L} D^{\alpha}{ }_{0^{+}} f(t)
$$

(ii) In case $\alpha=1,0<\beta<1$, and $b=0$, the Hilfer fractional derivative is identical to the classical Caputo derivative for $f$ of the form

$$
D_{0^{+}}^{1, \beta} f(t)=\mathcal{I}_{0^{+}}^{1-\beta} \frac{d}{d t} f(t)={ }^{c} D^{\beta}{ }_{0^{+}} f(t) .
$$

As of now, we characterize the abstract phase space $\mathscr{B}_{l}$, which is introduced in [51]. Let $g:(-\infty, 0] \rightarrow(0,+\infty)$ be a continuous function with $j=\int_{-\infty}^{0} g(t) d t<+\infty$. For each $i>0$, we define

$$
\mathscr{B}=\{\Phi:[-i, 0] \rightarrow Z, \Phi(t) \text { is bounded and measurable }\},
$$

and provide

$$
\|\Phi\|_{[-i, 0]}=\sup _{\gamma \in[-i, 0]}|\Phi(\gamma)| \quad \text { for every } \Phi \in \mathscr{B} .
$$


Define

$$
\mathscr{B}_{l}=\left\{\Phi:(-\infty, 0] \rightarrow Z ; \text { For every } i>0,\left.\Phi\right|_{[-i, 0]} \in \mathscr{B}, \int_{-\infty}^{0} g(t)\|\Phi\|_{[t, 0]} d t<+\infty\right\}
$$

and

$$
\|\Phi\|_{\mathcal{B}_{l}}=\int_{-\infty}^{0} g(t)\|\Phi\|_{[t, 0]} d t \quad \text { for every } \Phi \in \mathscr{B}_{l} .
$$

Hence $\left(\mathscr{B}_{l},\|\cdot\|_{\mathcal{B}_{l}}\right)$ is a Banach space. Suppose

$$
\mathscr{B}_{l}^{\prime}=\left\{\begin{array}{l}
z:(-\infty, b] \rightarrow Z \\
\left.z\right|_{\mathbb{N}} \in \mathcal{C}(\mathbb{N}, Z), \quad z(0)=\phi \in \mathscr{B}_{l},
\end{array}\right.
$$

fix $\|\cdot\|_{b}$ in $\mathscr{B}_{l}^{\prime}$, and it is characterized by

$$
\|z\|_{b}=\|\phi\|_{\mathscr{B}_{l}}+\sup \{|z(t)|: t \in(0, b]\}, \quad z \in \mathscr{B}_{l}^{\prime} .
$$

Lemma 2.5 ([64]) If $z \in \mathscr{B}_{l}^{\prime}$, then for $t \in \mathbb{N}, z_{t} \in \mathscr{B}_{l}$. Also,

$$
j|z(t)| \leq\left\|z_{t}\right\|_{\mathcal{B}_{l}} \leq\|\phi\|_{\mathcal{B}_{l}}+j \sup _{s \in[0, t]}|z(s)|
$$

where $j=\int_{-\infty}^{0} g(t) d t<+\infty$

Definition 2.6 ([65]) $z:(-\infty, b] \rightarrow Z$ is a mild solution of (1.1)-(1.2) only if $z(0)=\phi \in \mathscr{B}_{l}$ on $(-\infty, 0]$ and satisfies

$$
\begin{aligned}
z(t)= & \mathcal{J}^{-1} \mathcal{S}_{\alpha, \beta}(t) \mathcal{J} \phi \\
& +\int_{0}^{t}(t-s)^{\beta-1} \mathcal{J}^{-1} Q_{\beta}(t-s) f\left(s, z_{s}, \int_{0}^{s} e\left(s, \tau, z_{\tau}\right) d \tau\right) d s, \quad t \in \mathbb{N},
\end{aligned}
$$

where

$$
P_{\beta}(t)=t^{\beta-1} Q_{\beta}(t), \quad Q_{\beta}(t)=\int_{0}^{\infty} \beta \theta M_{\beta}(\theta) T\left(t^{\beta} \theta\right) d \theta .
$$

Remark 2.7 We define the mild solution of (1.1)-(1.2) as follows:

$$
M_{\beta}(\theta)=\sum_{k=1}^{\infty} \frac{(-\theta)^{k-1}}{(k-1) ! \Gamma(1-\beta k)}, \quad 0<\beta<1, \theta \in \mathcal{C},
$$

where $M_{\beta}(\theta)$ is a Wright function and satisfies

$$
\int_{0}^{\infty} \theta^{\iota} M_{\beta}(\theta) d \theta=\frac{\Gamma(1+\iota)}{\Gamma(1+\beta \iota)} \quad \text { for } \theta \geq 0
$$

Lemma $2.8([65])$ The operators $\mathcal{S}_{\alpha, \beta}(t)$ and $Q_{\beta}(t)$ satisfy the following conditions: 
- For $t \geq 0$, the operators $\mathcal{S}_{\alpha, \beta}(t)$ and $Q_{\beta}(t)$ are linearly bounded, i.e., for every $z \in Z$,

$$
\left\|\mathcal{S}_{\alpha, \beta}(t) z\right\| \leq \frac{M t^{\mu-1}}{\Gamma(\alpha(1-\beta)+\beta)}\|z\| ; \quad\left\|Q_{\beta}(t) z\right\| \leq \frac{M}{\Gamma(\beta)}\|z\|,
$$

where $\mathcal{S}_{\alpha, \beta}(t)=I_{0^{+}}^{\alpha(1-\beta)} P_{\beta}(t)$.

- The operators $\left\{\mathcal{S}_{\alpha, \beta}(t)\right\}_{t \geq 0}$ and $\left\{Q_{\beta}(t)\right\}_{t \geq 0}$ are strongly continuous.

Lemma 2.9 The strongly continuous operators $\left\{Q_{\beta}(t)\right\}_{t>0}$ and $\left\{\mathcal{S}_{\alpha, \beta}(t)\right\}_{t>0}, 0<t^{\prime}<t^{\prime \prime} \leq b$ are defined by

$$
\begin{aligned}
& \left\|\left(t^{\prime}\right)^{\beta-1} Q_{\beta}\left(t^{\prime}\right) z-\left(t^{\prime \prime}\right)^{\beta-1} Q_{\beta}\left(t^{\prime \prime}\right) z\right\| \rightarrow 0 \text { and } \\
& \left\|\mathcal{S}_{\alpha, \beta}\left(t^{\prime}\right) z-\mathcal{S}_{\alpha, \beta}\left(t^{\prime \prime}\right) z\right\| \rightarrow 0 \quad \text { as } t^{\prime \prime} \rightarrow t^{\prime} .
\end{aligned}
$$

Definition 2.10 ([60]) Assume $F^{+}$of the Banach space ( $F$ (positive cone), $\left.\leq\right)$. Define $\phi$ with values of $F^{+}$, it is said to be a measure of noncompactness on $Z$ iff $\phi(\overline{\mathrm{co}} Y)=\phi(Y)$ for $Y \subseteq Z$, where $\overline{\mathrm{co}} Y$ is a closed convex hull of $Y$.

The measure of noncompactness of $\phi$ is called:

(1) Monotone if and only if $\left(Y_{1} \subseteq Y_{2}\right) \Rightarrow\left(\phi\left(Y_{1}\right) \leq \phi\left(Y_{2}\right)\right), Y_{1}, Y_{2}$ are bounded subsets of $Z$;

(2) Nonsingular if and only if $\phi(\{a\} \cup Y)=\phi(Y)$ for every $a \in Z, Y \subset Z$;

(3) Regular if and only if $\phi(Y)=0, Y$ is relatively compact in $Z$.

The measure of noncompactness of Hausdorff $\widehat{\mu}$ is defined by

$$
\widehat{\mu}(Y)=\inf \left\{\epsilon>0 ; Y \subset \bigcup_{k=1}^{m} N_{k} \text { such that } \operatorname{diam}\left(N_{k}\right) \leq \epsilon\right\} .
$$

To know more information about the properties of MNC, the readers can refer to [66]. Now, for every $Y, Y_{1}, Y_{2}$ of $Z$,

(4) $\widehat{\mu}\left(Y_{1}+Y_{2}\right) \leq \widehat{\mu}\left(Y_{1}\right)+\widehat{\mu}\left(Y_{2}\right)$, where $Y_{1}+Y_{2}=\left\{y_{1}+y_{2}: y_{1} \in Y_{1}, y_{2} \in Y_{2}\right\}$;

(5) $\widehat{\mu}\left(Y_{1} \cup Y_{2}\right) \leq \max \left\{\widehat{\mu}\left(Y_{1}\right), \widehat{\mu}\left(Y_{2}\right)\right\}$;

(6) $\widehat{\mu}(\alpha Y) \leq|\alpha| \widehat{\mu}(Y)$ for any $\alpha \in \mathbb{R}$;

(7) If $Q: D(Q) \subseteq Z \rightarrow Y$, then $\widehat{\mu}_{Y}(Q Y) \leq k \widehat{\mu}(Y), Y \subseteq D(Q)$, here $Y$ is a Banach space and $k$ is any constant.

Lemma 2.11 ([66]) If $\mathcal{K} \subset \mathcal{C}(\mathbb{N}, Z)$ is bounded and equicontinuous, then $\widehat{\mu}(\mathcal{K}(t))$ is a continuous function for all $t \in \mathbb{N}$

$$
\widehat{\mu}(\mathcal{K})=\sup _{t \in \mathbb{N}}\{\widehat{\mu}(\mathcal{K}(t)), t \in \mathbb{N}\}, \quad \text { where } \mathcal{K}(t)=\{z(t): z \in \mathcal{K}\} \subseteq Z
$$

Theorem $2.12([62,67])$ If $\left\{u_{n}: \mathbb{N} \rightarrow Z\right\}$ is Bochner's integrable function with $\left\|u_{n}(t)\right\| \leq$ $\widehat{\mu}(t)$ a.e. for $t \in \mathbb{N}$ and for every $n \geq 1$, where $\widehat{\mu} \in L^{1}(\mathbb{N}, R)$, then $Y(t)=\widehat{\mu}\left(\left\{u_{n}(t): n \geq 1\right\}\right) \in$ $L^{1}(\mathbb{N}, R)$ and satisfies $\widehat{\mu}\left(\left\{\int_{0}^{t} u_{n}(s) d s: n \geq 1\right\}\right) \leq 2 \int_{0}^{t} \widehat{\mu}(s) d s$.

Lemma 2.13 ([68]) Let $K$ be a closed convex subset of $Z$ and $0 \in K$. If $F: K \rightarrow Z$ is a continuous map which satisfies Mönch's condition (i.e., $M \subseteq K$ is countable, $M \subseteq \overline{\mathrm{co}}(\{0\} \cup$ $F(M)) \Rightarrow \bar{M}$ is compact), then $F$ has a fixed point in $K$. 


\section{Existence}

In this section, we mainly focus on the existence of (1.1)-(1.2), and in order to prove the main theorem, we have the following assumptions.

$\left(\mathrm{A}_{0}\right)$ For all $K \subset Z, \theta \in(0, \infty)$ and $z \in K$,

$$
\left\|T\left(t_{2}^{\beta} \theta\right) z-T\left(t_{1}^{\beta} \theta\right) z\right\| \rightarrow 0, \quad \text { as } t_{2} \rightarrow t_{1} .
$$

$\left(\mathrm{A}_{1}\right)$ The function $f: \mathbb{N} \times \mathscr{B}_{l} \times Z \rightarrow Z$ satisfies the following:

(i) $f(\cdot, s, z)$ is measurable for every $(s, z) \in \mathscr{B}_{l} \times Z$ and $f(t, \cdot, \cdot)$ is continuous, $t \in \mathbb{N}$ and $z \in \mathscr{B}_{l}, f(\cdot, \cdot, z):(0, b] \rightarrow Z$ is strongly measurable.

(ii) There exist $\beta_{1} \in(0, \beta), m_{1} \in L^{\frac{1}{\beta_{1}}}\left(N, \mathbb{R}^{+}\right)$and $\Omega: \mathbb{R}^{+} \rightarrow \mathbb{R}^{+}$such that

$$
\|f(t, s, z)\| \leq m_{1}(t) \Omega\left(t^{1-v}\|s\|_{\mathcal{B}_{l}}+\|z\|\right)
$$

for every $t, s, z \in \mathbb{N} \times \mathscr{B}_{l} \times Z$, where $\widehat{\mu}$ satisfies $\liminf _{j \rightarrow \infty} \frac{\widehat{\mu}(j)}{j}=0$.

(iii) There exists $\beta_{2} \in(0, \beta), m_{2} \in L^{\frac{1}{\beta_{2}}}\left(\mathbb{N}, \mathbb{R}^{+}\right)$such that, for any $K_{1} \subset Z$ and $F_{1} \subset$ $\mathscr{B}_{l}$,

$$
\widehat{\mu}\left(f\left(t, F_{1}, K_{1}\right)\right) \leq m_{2}(t)\left[\sup _{-\infty<\xi \leq 0} \widehat{\mu}\left(F_{1}(\xi)\right)+\widehat{\mu}\left(K_{1}\right)\right] \text { for almost all } t \in \mathbb{N},
$$

where $F_{1}(\xi)=\left\{\grave{w}(\xi): \grave{w} \in K_{1}\right\}, \widehat{\mu}$ is the Hausdorff measure of noncompactness.

For $m_{i} \in L^{\frac{1}{\beta_{i}}}\left([0, b], \mathbb{R}^{+}\right), \beta_{i} \in(0, \beta), i=1,2$.

$\left(\mathrm{A}_{2}\right)$ The function $e: \mathbb{N} \times \mathbb{N} \times \mathscr{B}_{l} \rightarrow Z$ satisfies the following:

(i) $e(\cdot, s, z)$ is measurable for all $(s, z) \in \mathscr{B}_{l} \times Z$.

(ii) There exists $E_{0}>0$ such that $\|e(t, s, z)\| \leq E_{0}\left(1+\|z\|_{\mathscr{B}_{l}}\right)$ for every $t, z \in Z$, $s \in \mathscr{B}_{l}$.

(iii) There exists $m_{3} \in L^{1}\left(\mathbb{N}, \mathbb{R}^{+}\right)$such that, for any $K_{2} \subset Z$,

$$
\widehat{\mu}(f(t, s, z)) \leq m_{3}(t, s)\left[\sup _{-\infty<\xi \leq 0} \widehat{\mu}\left(K_{2}(\xi)\right)\right] \quad \text { for almost all } t \in \mathbb{N},
$$

with $m_{3}^{*}=\sup _{s \in \mathbb{N}} \int_{0}^{s} m_{3}(t, \tau) d \tau<\infty$.

For our convenience, we introduce

$$
\begin{aligned}
& M_{1}=k_{1}\left\|m_{1}\right\|_{L^{\frac{1}{\beta_{2}}}\left(\mathbb{N}, \mathbb{R}^{+}\right)}, \quad M_{2}=k_{2}\left\|m_{2}\right\|_{L^{\frac{1}{\beta_{2}}\left(\mathbb{N}, \mathbb{R}^{+}\right)},} \\
& k_{i}=\left[\left(\frac{1-\beta_{i}}{\beta-\beta_{i}}\right) b^{\left(\frac{\beta-\beta_{i}}{1-\beta_{i}}\right)}\right]^{1-\beta_{i}}, \quad \beta_{i} \in(0, \beta), i=1,2 .
\end{aligned}
$$

Theorem 3.1 Assume that $\left(A_{0}\right)-\left(A_{2}\right)$ are satisfied, then (1.1)-(1.2) has at least one mild solution if

$$
P^{*}=\frac{2 M M_{2} \widetilde{\mathcal{J}}_{m}^{1-v}\left(1+2 m_{3}^{*}\right)}{\Gamma(\beta)}<1 \text { for some } \frac{1}{2}<\beta<1
$$


Proof We define the operator $\Upsilon: \mathscr{B}_{l}^{\prime} \rightarrow \mathscr{B}_{l}^{\prime}$ by

$$
\Upsilon z(t)=\left\{\begin{array}{lr}
\phi(t), & t \in(-\infty, 0], \\
\mathcal{J}^{-1} \mathcal{S}_{\alpha, \beta}(t) \mathcal{J} \phi & \\
\quad+\int_{0}^{t}(t-s)^{\beta-1} \mathcal{J}^{-1} Q_{\beta}(t-s) f\left(s, z_{s}, \int_{0}^{s} e\left(s, \tau, z_{\tau}\right) d \tau\right) d s, & t \in \mathbb{N} .
\end{array}\right.
$$

For $\phi \in \mathscr{B}_{l}$, we define $\hat{\eta}$ as follows:

$$
\hat{\eta}(t)= \begin{cases}\phi(t), & t \in(-\infty, 0], \\ \mathcal{J}^{-1} \mathcal{S}_{\alpha, \beta}(t) \mathcal{J} \phi, & t \in \mathbb{N},\end{cases}
$$

then $\hat{\eta} \in \mathscr{B}_{l}^{\prime}$. Let $z(t)=g(t)+\hat{\eta}(t),-\infty<t \leq b$. Clearly, $z$ satisfies (2.1) if and only if $g$ satisfies $g_{0}=0$ and

$$
g(t)=\int_{0}^{t}(t-s)^{\beta-1} \mathcal{J}^{-1} Q_{\beta}(t-s) f\left(s, g_{s}+\hat{\eta}_{s}, \int_{0}^{s} e\left(s, \tau, g_{\tau}+\hat{\eta}_{\tau}\right) d \tau\right) d s .
$$

Let $\mathscr{B}_{l}^{\prime \prime}=\left\{g \in \mathscr{B}_{l}^{\prime}: g_{0}=0 \in \mathscr{B}_{l}\right\}$. For any $g \in \mathscr{B}_{l}^{\prime \prime}$,

$$
\begin{aligned}
\|g\|_{b} & =\left\|g_{0}\right\|_{\mathcal{B}_{l}}+\sup \{\|g(s)\|: 0 \leq s \leq b\} \\
& =\sup \{\|g(s)\|: 0 \leq s \leq b\} .
\end{aligned}
$$

Hence $\left(\mathscr{B}_{l}^{\prime \prime},\|\cdot\|_{b}\right)$ is a Banach space. Now $\ell>0$, we fix $F_{\ell}=\left\{g \in \mathscr{B}_{l}^{\prime \prime}:\|g\|_{b} \leq \ell\right\}$, then $F_{\ell} \subseteq \mathscr{B}_{l}^{\prime \prime}$ is uniformly bounded, $g \in F_{\ell}$, and referring to Lemma 2.5 ,

$$
\begin{aligned}
\left\|g_{t}+\hat{\eta}_{t}\right\|_{\mathcal{B}_{l}} & \leq\left\|g_{t}\right\|_{\mathcal{B}_{l}}+\left\|\hat{\eta}_{t}\right\|_{\mathcal{B}_{l}} \\
& \leq j\left(\ell+\widetilde{\mathcal{J}}_{m} \frac{M t^{\mu-1}}{\Gamma(\alpha(1-\beta)+\beta)} \mathcal{J}_{m}|\phi|\right)+\|\phi\|_{\mathcal{B}_{l}}=\ell^{\prime} .
\end{aligned}
$$

We define the operator $\tilde{\Upsilon}: \mathscr{B}_{l}^{\prime \prime} \rightarrow \mathcal{B}_{l}^{\prime \prime}$ as follows:

$$
\tilde{\Upsilon} g(t)= \begin{cases}0, & t \in(-\infty, 0], \\ \int_{0}^{t}(t-s)^{\beta-1} \mathcal{J}^{-1} Q_{\beta}(t-s) & \\ \quad \times f\left(s, g_{t}+\hat{\eta}_{t}, \int_{0}^{s} e\left(s, \tau, g_{\tau}+\hat{\eta}_{\tau}\right) d \tau\right) d s, & t \in \mathbb{N} .\end{cases}
$$

To prove that $\widetilde{\Upsilon}$ has a fixed point.

Now we divide the proof into a few steps for our benefit.

Step 1: For $\ell>0, \widetilde{\Upsilon}\left(F_{\ell}\right) \subseteq F_{\ell}$. If it is false, then $g^{\ell}(\cdot) \in F_{\ell}$ and $t \in \mathbb{N}$ such that $\left\|\left(\widetilde{\Upsilon} g^{\ell}\right)(t)\right\|>$ $\ell$. Suppose $\ell>0$, and consider $\left\{F_{\ell}=z \in \mathcal{C}_{1-v}:\|z\|_{v} \leq \ell\right\}$. It is understood that $F_{\ell}$ is a closed, bounded, and convex set of $C$. Furthermore, from Lemma 2.8, ( $\left.\mathrm{A}_{1}\right)$, and Hölder's inequality, we have

$$
\begin{aligned}
& \ell<\sup _{t \in \mathbb{N}} t^{1-\nu}\left\|\left(\widetilde{\Upsilon} g^{\ell}\right)(t)\right\| \\
& \quad \leq b^{1-v}\left\|\int_{0}^{t}(t-s)^{\beta-1} \mathcal{J}^{-1} Q_{\beta}(t-s) f\left(s, g_{s}^{\ell}+\hat{\eta}_{s}, \int_{0}^{s} e\left(s, \tau, g_{\tau}^{\ell}+\hat{\eta}_{\tau}\right) d \tau\right) d s\right\|
\end{aligned}
$$




$$
\begin{aligned}
& \leq b^{1-\nu}\left\|\int_{0}^{t}(t-s)^{\beta-1} \mathcal{J}^{-1} Q_{\beta}(t-s) m_{1}(s) \Omega\left(\ell^{\prime}+b E_{0}\left(1+\ell^{\prime}\right)\right) d s\right\| \\
& \leq b^{1-\nu} \frac{M M_{1} \widetilde{\mathcal{J}}_{m}}{\Gamma(1+\beta)} \Omega\left(\ell^{\prime}+b E_{0}\left(1+\ell^{\prime}\right)\right) .
\end{aligned}
$$

We now divide (3.5) by $\ell$, and taking $\ell \rightarrow \infty$, it contradicts with (3.1). Therefore, $\widetilde{\Upsilon}\left(F_{\ell}\right) \subseteq$ $F_{\ell}$.

Step 2: $\widetilde{\Upsilon}$ is continuous on $F_{\ell}$.

For all $g^{m}, g \in F_{\ell}(\mathbb{N}), m=0,1,2, \ldots$, with $\lim _{m \rightarrow \infty} g^{m}=g$, then we have $\lim _{m \rightarrow \infty} g^{m}(t)=$ $g(t)$ and

$$
\lim _{m \rightarrow \infty} t^{1-v} g^{m}(t)=t^{1-v} g(t)
$$

Consider $f\left(t, t^{1-v} z^{m}(t), \int_{0}^{t} e\left(t, s, s^{1-v} z^{m}(s)\right) d s\right)$, and we take

$$
\begin{aligned}
& F_{m}(s)=f\left(s, s^{1-v}\left(g_{s}^{m}+\hat{\eta}_{s}\right), \int_{0}^{s} e\left(s, \varepsilon, \varepsilon^{1-v}\left(g_{\varepsilon}^{m}+\hat{\eta}_{\varepsilon}\right)\right) d \varepsilon\right) \text { and } \\
& F(s)=f\left(s, s^{1-v}\left(g_{s}+\hat{\eta}_{s}\right), \int_{0}^{s} e\left(s, \varepsilon, \varepsilon^{1-v}\left(g_{\varepsilon}+\hat{\eta}_{\varepsilon}\right)\right) d \varepsilon\right) .
\end{aligned}
$$

Lebesgue's dominated convergence theorem and hypotheses $\left(\mathrm{A}_{1}\right),\left(\mathrm{A}_{2}\right)$ give

$$
\int_{0}^{t}(t-s)^{\beta-1} \mathcal{J}^{-1} Q_{\beta}(t-s)\left\|F_{m}(s)-F(s)\right\| d s \rightarrow 0 \quad \text { as } m \rightarrow \infty, t \in \mathbb{N} .
$$

Now, by $\left(A_{1}\right)$, we have

$$
\left\|\tilde{\Upsilon} g^{m}-\tilde{\Upsilon} g\right\|_{\mathcal{C}} \leq \frac{b^{1-v} M \widetilde{\mathcal{J}}_{m}}{\Gamma(\beta)} \int_{0}^{t}(t-s)^{\beta-1}\left\|F_{m}(s)-F(s)\right\| d s .
$$

Using (3.6) in (3.7), we get

$$
\left\|\tilde{\Upsilon} g^{m}-\tilde{\Upsilon} g\right\|_{\mathcal{C}} \rightarrow 0 \quad \text { as } m \rightarrow \infty
$$

therefore, $\tilde{\Upsilon}$ is continuous on $F_{\ell}$.

Step 3: $\widetilde{\Upsilon}\left(F_{\ell}\right)$ is equicontinuous on $\mathbb{N}$. Let $\gamma \in \widetilde{\Upsilon}\left(F_{\ell}\right)$.

For $0<t_{1}<t_{2}<b$, we have

$$
\begin{aligned}
\left\|\gamma\left(t_{2}\right)-\gamma\left(t_{1}\right)\right\|= & \| \mathcal{J}^{-1} \int_{0}^{t_{2}} t_{2}^{1-v}\left(t_{2}-s\right)^{\beta-1} Q_{\beta}\left(t_{2}-s\right) F(s) d s \\
& -\mathcal{J}^{-1} \int_{0}^{t_{1}} t_{1}^{1-v}\left(t_{1}-s\right)^{\beta-1} Q_{\beta}\left(t_{1}-s\right) F(s) d s \| \\
\leq & \left\|\int_{t_{1}}^{t_{2}} t_{2}^{1-v}\left(t_{2}-s\right)^{\beta-1} \mathcal{J}^{-1} Q_{\beta}\left(t_{1}-s\right) F(s) d s\right\| \\
& +\left\|\int_{0}^{t_{1}}\left[t_{2}^{1-v}\left(t_{2}-s\right)^{\beta-1}-t_{1}^{1-v}\left(t_{1}-s\right)^{\beta-1}\right] \mathcal{J}^{-1} Q_{\beta}\left(t_{2}-s\right) F(s) d s\right\| \\
& +\left\|\int_{0}^{t_{1}} t_{1}^{1-v}\left(t_{1}-s\right)^{\beta-1} \mathcal{J}^{-1}\left[Q_{\beta}\left(t_{2}-s\right)-Q_{\beta}\left(t_{1}-s\right)\right] F(s) d s\right\|
\end{aligned}
$$


Kavitha et al. Advances in Difference Equations

(2021) 2021:467

Page 9 of 18

$$
\begin{aligned}
\leq & \frac{M \widetilde{\mathcal{J}}_{m}}{\Gamma(\beta)} \Omega\left(\ell^{\prime}+b E_{0}\left(1+\ell^{\prime}\right)\right)\left\|\int_{t_{1}}^{t_{2}} t_{2}^{1-v}\left(t_{2}-s\right)^{\beta-1} m_{1}(s) d s\right\| \\
& +\frac{2 M \widetilde{\mathcal{J}}_{m}}{\Gamma(\beta)}\left\|\int_{0}^{t_{1}}\left[t_{2}^{1-v}\left(t_{2}-s\right)^{\beta-1}-t_{1}^{1-v}\left(t_{1}-s\right)^{\beta-1}\right] m_{1}(s) d s\right\| \\
& +\left\|\int_{0}^{t_{1}} t_{1}^{1-v}\left(t_{1}-s\right)^{\beta-1} \mathcal{J}^{-1}\left[Q_{\beta}\left(t_{2}-s\right)-Q_{\beta}\left(t_{1}-s\right)\right] F(s) d s\right\| .
\end{aligned}
$$

By Lemma 2.9 and Lebesgue's integral dominance convergence theorem, we get $\| \gamma\left(t_{2}\right)-$ $\gamma\left(t_{1}\right) \|$ becomes zero as $t_{2}-t_{1} \rightarrow 0$.

Thus, $\tilde{\Upsilon}\left(F_{\ell}\right)$ is equicontinuous on $\mathbb{N}$.

Step 4: Mönch's condition: Assume that $\mathcal{K} \subseteq F_{\ell}$ is countable and $\mathcal{K} \subseteq \operatorname{conv}(\{0\} \cup \tilde{\Upsilon}(\mathcal{K}))$. To prove $\widehat{\mu}(\mathcal{K})=0$, where $\widehat{\mu}$ is the Hausdorff measure of noncompactness. If $\mathcal{K}=\left\{g^{m}\right\}_{m=1}^{\infty}$, thus we show that $\widetilde{\Upsilon}(\mathcal{K})(t)$ is relatively compact in $Z$ for all $t \in \mathbb{N}$. By using Theorem 2.12,

$$
\begin{aligned}
& \widehat{\mu}\left(\left\{\left(\widetilde{\Upsilon} g^{m}\right)(t)\right\}_{m=1}^{\infty}\right) \\
& =\widehat{\mu}\left(\left\{t^{1-v} \int_{0}^{t}(t-s)^{\beta-1} \mathcal{J}^{-1} Q_{\beta}(t-s) F_{m}(s)\right\}_{m=1}^{\infty}\right) \\
& \leq \frac{2 M \widetilde{\mathcal{J}}_{m} b^{1-v}}{\Gamma(\beta)} \int_{0}^{t}(t-s)^{\beta-1} \widehat{\mu}\left(\left\{F_{m}(s)\right\}_{m=1}^{\infty}\right) d s \\
& \leq \frac{2 M \widetilde{\mathcal{J}}_{m} b^{1-v}}{\Gamma(\beta)} \int_{0}^{t}(t-s)^{\beta-1} m_{2}(s)\left[\sup _{-\infty<\tau \leq 0} \&\left(\left\{g^{m}(s+\tau)+\hat{\eta}(s+\tau)\right\}_{m=1}^{\infty}\right)\right. \\
& \left.+\widehat{\mu}\left(\left\{\int_{0}^{s} e\left(s, \varepsilon, g_{\varepsilon}^{m}+\hat{\eta}_{\varepsilon}\right) d \varepsilon\right\}_{m=1}^{\infty}\right)\right] d s \\
& \leq \frac{2 M \widetilde{\mathcal{J}}_{m} b^{1-v}}{\Gamma(\beta)} \int_{0}^{t}(t-s)^{\beta-1} m_{2}(s) \sup _{-\infty<\tau \leq s} \widehat{\mu}\left(\left\{g^{m}(\tau)+\hat{\eta}(\tau)\right\}_{m=1}^{\infty}\right) d s \\
& +\frac{4 M \widetilde{\mathcal{J}}_{m} b^{1-v}}{\Gamma(\beta)} \int_{0}^{t}(t-s)^{\beta-1} m_{2}(s) \int_{0}^{s} \widehat{\mu}\left(\left\{e\left(s, \varepsilon, g_{\varepsilon}^{m}+\hat{\eta}_{\varepsilon}\right) d \varepsilon\right\}_{m=1}^{\infty}\right) d s \\
& \leq \frac{2 M \widetilde{\mathcal{J}}_{m} b^{1-v}}{\Gamma(\beta)} \int_{0}^{t}(t-s)^{\beta-1} m_{2}(s) \sup _{-\infty<\tau \leq s} \widehat{\mu}\left(\left\{g^{m}(\tau)+\hat{\eta}(\tau)\right\}_{m=1}^{\infty}\right) d s \\
& +\frac{4 M \widetilde{\mathcal{J}}_{m} b^{1-v}}{\Gamma(\beta)} \int_{0}^{t}(t-s)^{\beta-1} m_{2}(s) \int_{0}^{s} m_{3}(s, \varepsilon)\left[\sup _{-\infty<\xi \leq 0} \widehat{\mu}\left(\left(g_{\varepsilon}^{m}+\hat{\eta}_{\varepsilon}\right)(\xi)\right) d \varepsilon\right] d s \\
& \leq \frac{2 M M_{2} \widetilde{\mathcal{J}}_{m} c^{1-v}}{\Gamma(\beta)} \sup _{-\infty<\tau \leq s} \widehat{\mu}\left(\left\{g^{m}(\tau)+\hat{\eta}(\tau)\right\}_{m=1}^{\infty}\right) \\
& +\frac{4 M M_{2} \widetilde{\mathcal{J}}_{m} b^{1-v}}{\Gamma(\beta)} \int_{0}^{s} m_{3}(s, \varepsilon)\left[\sup _{-\infty<\xi \leq 0} \widehat{\mu}\left(\left(g_{\varepsilon}^{m}+\hat{\eta}_{\varepsilon}\right)(\xi)\right) d \varepsilon\right] \\
& \leq \frac{2 M M_{2} \tilde{\mathcal{J}}_{m} b^{1-v}}{\Gamma(\beta)}\left[1+2 m_{3}^{*}\right] \sup _{0 \leq \tau \leq s} \widehat{\mu}\left(\left\{g^{m}(\tau)+\hat{\eta}(\tau)\right\}_{m=1}^{\infty}\right) \\
& \leq \frac{2 M M_{2} \widetilde{\mathcal{J}}_{m} b^{1-v}}{\Gamma(\beta)}\left[1+2 m_{3}^{*}\right] \sup _{0 \leq \tau \leq s} \widehat{\mu}(\mathcal{K}(\tau)) \text {. }
\end{aligned}
$$

That is,

$$
\widehat{\mu}(\widetilde{\Upsilon} \mathcal{K}(t)) \leq \frac{2 M M_{2} \widetilde{\mathcal{J}}_{m} b^{1-v}\left(1+2 m_{3}^{*}\right)}{\Gamma(\beta)} \sup _{0 \leq \tau \leq s} \widehat{\mu}(\mathcal{K}(\tau)) .
$$


Therefore, by using Lemma 2.11,

$$
\widehat{\mu}(\mathcal{K}) \leq \widehat{\mu}\left(\operatorname{conv}(\{0\} \cup(\widetilde{\Upsilon}(\mathcal{K})))=\widehat{\mu}(\widetilde{\Upsilon}(\mathcal{K})) \leq P^{*} \widehat{\mu}(\mathcal{K}),\right.
$$

where $P^{*}$ is defined in (3.1), hence $\widehat{\mu}(\mathcal{K})=0$.

Lemma 2.13 shows that $\widetilde{\Upsilon}$ has a fixed point $\mathcal{K} \in F_{\ell}$. Consequently, $z=g+\hat{\eta}$ is a mild solution of (1.1)-(1.2). The proof is now completed.

\section{Controllability}

In this section, we mainly focus on the controllability of (1.3)-(1.4). So, we now introduce the mild solution of (1.3)-(1.4) as follows.

Definition 4.1 A function $z:(-\infty, b] \rightarrow Z$ is said to be a mild solution of (1.1)-(1.2) if and only if $z(0)=\phi \in \mathscr{B}_{l}$ on $(-\infty, 0]$ and $z$ satisfies

$$
\begin{aligned}
z(t)= & \mathcal{J}^{-1} \mathcal{S}_{\alpha, \beta}(t) \mathcal{J} \phi+\int_{0}^{t}(t-s)^{\beta-1} \mathcal{J}^{-1} Q_{\beta}(t-s) f\left(s, z_{s}, \int_{0}^{s} e\left(s, \tau, z_{\tau}\right) d \tau\right) d s \\
& +\int_{0}^{t}(t-s)^{\beta-1} \mathcal{J}^{-1} Q_{\beta}(t-s) B u_{z}(s) d s, \quad t \in \mathbb{N} .
\end{aligned}
$$

Lemma 4.2 System (1.3)-(1.4) is said to be controllable on $\mathbb{N}$ if, for every $\phi \in \mathscr{B}_{l}, z^{1} \in Z$, there exists $u \in L^{2}(\mathbb{N}, U)$ such that $z(t)$ of (1.3)-(1.4) satisfies $z(b)=z^{1}$.

Controllability results are proved in relation to the following hypotheses:

$\left(\mathrm{A}_{3}\right)$ The operator $B: L^{2}(\mathbb{N}, \mathbb{U}) \rightarrow L^{1}(\mathbb{N}, Z)$ which is bounded, $W: L^{2}(\mathbb{N}, \mathbb{U}) \rightarrow Z$ defined by

$$
W u=\int_{0}^{b}(b-s)^{\beta-1} \mathcal{J}^{-1} Q_{\beta}(t-s) B u(s) d s,
$$

satisfies:

(i) $W^{-1}$ takes the value in $L^{2}((0, b], \mathbb{U}) / \operatorname{Ker} W$, there exist $M_{b}>0, M_{w}>0$ such that $\|B\| \leq M_{b}$ and $\left\|W^{-1}\right\| \leq M_{w}$.

(ii) There exists $\beta_{4} \in(0, \beta)$, and for every $K \in Z, m_{4} \in L^{\frac{1}{\beta_{4}}}\left((0, b], \mathbb{R}^{+}\right)$such that $\widehat{\mu}\left(\left(W^{-1} K\right)(t)\right) \leq m_{4}(t) \widehat{\mu}(K)$. Here, $m_{i} \in L^{\frac{1}{\beta_{i}}}\left([0, b], \mathbb{R}^{+}\right), \beta_{i} \in(0, \beta), i=1,2,3,4$.

For our convenience, we introduce

$$
\begin{aligned}
& M_{4}=k_{4}\left\|m_{4}\right\|_{L^{\frac{1}{\beta_{4}}}\left(\mathbb{N}, \mathbb{R}^{+}\right)}, \quad C^{*}=\sqrt{\frac{b^{2 \beta-1}}{2 \beta-1}}, \\
& k_{i}=\left[\left(\frac{1-\beta_{i}}{\beta-\beta_{i}}\right) b^{\left.\frac{\left(\beta-\beta_{i}\right.}{1-\beta_{i}}\right)}\right]^{1-\beta_{i}}, \quad i=1,2,3,4 .
\end{aligned}
$$

Theorem 4.3 Assume that $\left(A_{0}\right)-\left(A_{3}\right)$ are satisfied, then (1.3)-(1.4) is controllable on $(0, b]$ if

$$
\frac{2 M \widetilde{\mathcal{J}}_{m} M_{2} b^{1-v}\left(1+2 m_{3}^{*}\right)}{\Gamma(\beta)}\left[1+\frac{2 M M_{b} M_{4} \widetilde{\mathcal{J}}_{m}}{\Gamma(\beta)}\right] \widehat{\mu}(\mathcal{K}(\tau))<1 \quad \text { for some } \frac{1}{2}<\beta<1 .
$$


Proof By using $\left(\mathrm{A}_{3}\right)$, we define $u_{z}(t)$ by

$$
\begin{aligned}
u_{z}(t)= & W^{-1}\left[z^{1}-\mathcal{J}^{-1} \mathcal{S}_{\alpha, \beta}(t) \mathcal{J} \phi\right. \\
& \left.-\int_{0}^{b}(b-s)^{\beta-1} \mathcal{J}^{-1} Q_{\beta}(b-s) f\left(s, g_{s}, \int_{0}^{s} e\left(s, \tau, g_{\tau}\right) d \tau\right) d s\right](t) .
\end{aligned}
$$

Let $\Upsilon: \mathscr{B}_{l}^{\prime} \rightarrow \mathscr{B}_{l}^{\prime}$ be defined by

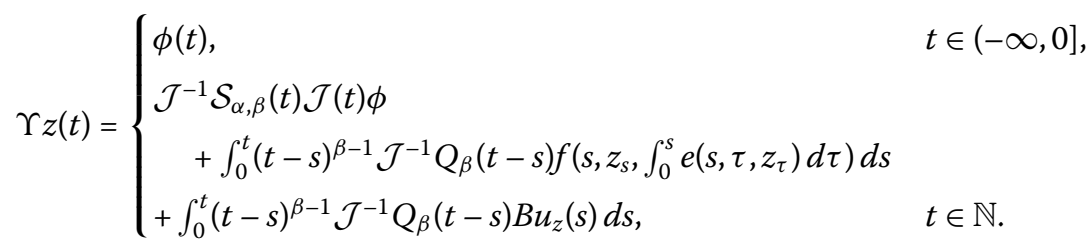

For $\phi \in \mathcal{B}_{l}$, we have

$$
\hat{\eta}(t)= \begin{cases}\phi(t), & t \in(-\infty, 0] \\ \mathcal{J}^{-1} \mathcal{S}_{\alpha, \beta}(t) \mathcal{J} \phi, & t \in \mathbb{N}\end{cases}
$$

then $\hat{\eta} \in \mathscr{B}_{l}^{\prime}$. Let $z(t)=g(t)+\hat{\eta}(t),-\infty<t \leq b$. Now, we identified that $z$ satisfies (4.1) if and only if $g$ satisfies $g_{0}=0$ and

$$
\begin{aligned}
g(t)= & \int_{0}^{t}(t-s)^{\beta-1} \mathcal{J}^{-1} Q_{\beta}(t-s) f\left(s, g_{s}+\hat{\eta}_{s}, \int_{0}^{s} e\left(s, \tau, g_{\tau}+\hat{\eta}_{\tau}\right) d \tau\right) d s \\
& +\int_{0}^{t}(t-s)^{\beta-1} \mathcal{J}^{-1} Q_{\beta}(t-s) B u_{z}(s) d s,
\end{aligned}
$$

where

$$
\begin{aligned}
u_{z}(s)= & W^{-1}\left[z^{1}-\mathcal{J}^{-1} \mathcal{S}_{\alpha, \beta}(t) \mathcal{J} \phi-\int_{0}^{b}(b-s)^{\beta-1} \mathcal{J}^{-1} Q_{\beta}(b-s)\right. \\
& \left.\times f\left(s, g_{s}+\hat{\eta}_{s}, \int_{0}^{s} e\left(s, \tau, g_{\tau}+\hat{\eta}_{\tau}\right) d \tau\right) d s\right](t) .
\end{aligned}
$$

We define the operator $\tilde{\Upsilon}: \mathcal{B}_{h}^{\prime \prime} \rightarrow \mathcal{B}_{h}^{\prime \prime}$ by

$$
\tilde{\Upsilon} g(t)= \begin{cases}0, & t \in(-\infty, 0] \\ \int_{0}^{t}(t-s)^{\beta-1} \mathcal{J}^{-1} Q_{\beta}(t-s) f\left(s, g_{s}+\hat{\eta}_{s}, \int_{0}^{s} e\left(s, \tau, g_{\tau}+\hat{\eta}_{\tau}\right) d \tau\right) d s & \\ \quad+\int_{0}^{t}(t-s)^{\beta-1} \mathcal{J}^{-1} Q_{\beta}(t-s) B u_{z}(s) d s, & t \in \mathbb{N} .\end{cases}
$$

Now, to show $\widetilde{\Upsilon}$ has a fixed point. We divide the proof into the following steps for our convenience.

Step 1: To prove that there exists a constant $\ell>0$ such that $\widetilde{\Upsilon}\left(F_{\ell}\right) \subseteq F_{\ell}$. If it fails, then $g^{\ell}(\cdot) \in F_{\ell}$ and $t \in \mathbb{N}$ such that $\left\|\widetilde{\Upsilon}\left(g^{\ell}\right)(t)\right\|>\ell$.

Take $\ell>0$ and consider $\left\{F_{\ell}=z \in \mathcal{C}_{1-v}:\|z\|_{v} \leq \ell\right\}$. Apparently, $F_{\ell}$ is a closed, bounded, and convex set of $C$. 
Using Lemma 2.8, $\left(\mathrm{A}_{1}\right),\left(\mathrm{A}_{3}\right)$, and Holder's inequality, we have

$$
\begin{aligned}
& \ell<\sup _{x \in \mathbb{N}} t^{1-v}\left\|\left(\widetilde{\Upsilon} g^{\ell}\right)(t)\right\| \\
& \leq b^{1-v}\left\|\int_{0}^{t}(t-s)^{\beta-1} \mathcal{J}^{-1} Q_{\beta}(t-s) f\left(s, g_{s}+\hat{\eta}_{s}, \int_{0}^{s} e\left(s, \tau, g_{\tau}+\hat{\eta}_{\tau}\right) d \tau\right) d s\right\| \\
& +b^{1-v}\left\|\int_{0}^{t}(t-s)^{\beta-1} \mathcal{J}^{-1} Q_{\beta}(t-s) B u_{g^{\ell}}(s) d s\right\|, \quad t \in \mathbb{N} \\
& \leq \frac{M \widetilde{\mathcal{J}}_{m} b^{1-v}}{\Gamma(\beta)}\left\|\int_{0}^{t}(t-s)^{\beta-1} m_{1}(s) \Omega\left(\ell^{\prime}+b E_{0}\left(1+\ell^{\prime}\right)\right) d s\right\| \\
& +\frac{M M_{b} \tilde{\mathcal{J}}_{m} b^{1-v}}{\Gamma(\beta)} \sqrt{\frac{b^{2 \beta-1}}{2 \beta-1}}\left\|u_{g} \ell\right\|_{L^{2}} \\
& \leq \frac{M M_{1} \tilde{\mathcal{J}}_{m} b^{1-v}}{\Gamma(\beta)} \Omega\left(\ell^{\prime}+b E_{0}\left(1+\ell^{\prime}\right)\right) \\
& +\frac{M M_{b} M_{w} \tilde{\mathcal{J}}_{m} b^{1-v}}{\Gamma(\beta)} \sqrt{\frac{b^{2 \beta-1}}{2 \beta-1}}\left[\left\|z^{1}\right\|+\mathcal{J}^{-1} \mathcal{S}_{\alpha, \beta}(t)(b) \mathcal{J} \phi\right. \\
& \left.+\int_{0}^{b}(b-\gamma)^{\beta-1} \mathcal{J}^{-1} Q_{\beta}(t-\gamma) f\left(\gamma, g_{\gamma}+\hat{\eta}_{\gamma}, \int_{0}^{\gamma} e\left(\gamma, \tau, g_{\tau}+\hat{\eta}_{\tau}\right) d \tau\right) d \gamma\right] \\
& \leq \frac{M M_{1} \tilde{\mathcal{J}}_{m} b^{1-v}}{\Gamma(\beta)} \Omega\left(\ell^{\prime}+b E_{0}\left(1+\ell^{\prime}\right)\right)+\frac{M M_{b} M_{w} \widetilde{\mathcal{J}}_{m}}{\Gamma(\beta)} C^{*}\left[b^{1-v}\left\|z^{1}\right\|\right. \\
& \left.+\frac{\widetilde{\mathcal{J}}_{m} M \mathcal{J}_{m}}{\Gamma(\gamma(1-\beta)+\beta)}\|\phi\|+\frac{M M_{1} \widetilde{\mathcal{J}}_{m} b^{1-\nu}}{\Gamma(\beta)} \Omega\left(\ell^{\prime}+b E_{0}\left(1+\ell^{\prime}\right)\right)\right] \\
& \leq \frac{M M_{1} \tilde{\mathcal{J}}_{m} b^{1-v}}{\Gamma(\beta)} \Omega\left(\ell^{\prime}+b E_{0}\left(1+\ell^{\prime}\right)\right)\left[1+\frac{M \widetilde{\mathcal{J}}_{m} M_{b} M_{w}}{\Gamma(\beta)} C^{*}\right] \\
& +\frac{M \widetilde{\mathcal{J}}_{m} M_{b} M_{w}}{\Gamma(\beta)} C^{*}\left[b^{1-v}\left\|z^{1}\right\|+\frac{\widetilde{\mathcal{J}}_{m} M \mathcal{J}_{m}}{\Gamma(\gamma(1-\beta)+\beta)}\|\phi\|\right]
\end{aligned}
$$

Take $\rho=\ell^{\prime}+b E_{0}\left(1+\ell^{\prime}\right)$, note that $\rho \rightarrow \infty$ as $\ell \rightarrow \infty$.

Dividing (4.5) by $\ell$ and taking $\ell \rightarrow \infty$, we have

$$
1 \leq \frac{M M_{1} \widetilde{\mathcal{J}}_{m} b^{1-v}}{\Gamma(\beta)} \lim _{\rho \rightarrow \infty} \inf \frac{\widehat{\mu}(\rho)}{\rho} \frac{\rho}{\ell}\left[1+\frac{M \widetilde{\mathcal{J}}_{m} M_{b} M_{w}}{\Gamma(\beta)} C^{*}\right]
$$

then by $\left(\mathrm{A}_{1}\right)(i i),(4.6)$ is a contradiction. Hence $\widetilde{\Upsilon}\left(F_{\ell}\right) \subseteq F_{\ell}$.

Step 2: Similar to Step 2 of Theorem 3.1.

Step 3: For $g \in F_{\ell}$, assume $g(t)=t^{\nu-1} \Upsilon z(t), \widetilde{\Upsilon}$ provides bounded sets into equicontinuous sets of $C$ for all $y \in F_{\ell}$, there exists $\Upsilon \in \widetilde{\Upsilon}(z)$ such that $\left\|\Upsilon z\left(t_{2}\right)-\Upsilon z\left(t_{1}\right)\right\| \rightarrow 0$ as $t_{2} \rightarrow t_{1}$.

$$
\begin{aligned}
\Upsilon z(t)= & \mathcal{J}^{-1} \mathcal{S}_{\alpha, \beta}(t) \mathcal{J}(t) \phi+\int_{0}^{t}(t-s)^{\beta-1} \mathcal{J}^{-1} Q_{\beta}(t-s) \\
& (\times) f\left(s, g_{s}+\hat{\eta}_{s}, \int_{0}^{s} e\left(s, \tau, z_{\tau}\right) d \tau\right) d s \\
& +\int_{0}^{t}(t-s)^{\beta-1} \mathcal{J}^{-1} Q_{\beta}(t-s) B u_{g}(s) d s .
\end{aligned}
$$


Hereafter, we continue our proof as per Step 3 of Theorem 3.1, and hence $\widetilde{\Upsilon}\left(F_{\ell}\right)$ is equicontinuous.

Step 4: Mönch's condition: Consider $\mathcal{K} \subseteq F_{\ell}$ is countable and $\mathcal{K} \subseteq \operatorname{conv}(\{0\} \cup \widetilde{\Upsilon}(\mathcal{K}))$. To prove $\widehat{\mu}(\mathcal{K})=0$, here $\widehat{\mu}$ is the Hausdorff measure of noncompactness. If $\mathcal{K}=\left\{g^{m}\right\}_{m=1}^{\infty}$, then we show that $\tilde{\Upsilon}(\mathcal{K})(t)$ is relatively compact in $Z$ for all $t \in \mathbb{N}$. By Theorem 2.12, we obtain

$$
\begin{aligned}
\widehat{\mu}\left(\left\{\left(\widetilde{\Upsilon} g^{m}\right)(t)\right\}_{m=1}^{\infty}\right)= & \widehat{\mu}\left(\left\{\int_{0}^{t}(t-s)^{\beta-1} \mathcal{J}^{-1} Q_{\beta}(t-s)\left[F_{m}(s)+B u_{g^{m}}(s)\right]\right\}_{m=1}^{\infty}\right) \\
\leq & \frac{2 M \widetilde{\mathcal{J}}_{m} b^{1-v}}{\Gamma(\beta)} \int_{0}^{t}(t-s)^{\beta-1} \widehat{\mu}\left(\left\{F_{m}(s)\right\}_{m=1}^{\infty}\right) d s \\
& +\frac{2 M M_{b} \widetilde{\mathcal{J}}_{m} b^{1-v}}{\Gamma(\beta)} \int_{0}^{t}(t-s)^{\beta-1} \widehat{\mu}\left(\left\{u_{g^{m}}(s)\right\}_{m=1}^{\infty}\right) d s \\
\leq & \mathbb{I}_{1}+\mathbb{I}_{2},
\end{aligned}
$$

where

$$
\begin{aligned}
& \mathbb{I}_{1}=\frac{2 M \widetilde{\mathcal{J}}_{m} b^{1-v}}{\Gamma(\beta)} \int_{0}^{t}(t-s)^{\beta-1} \widehat{\mu}\left(\left\{F_{m}(s)\right\}_{m=1}^{\infty}\right) d s \\
& \leq \frac{2 M M_{2} \widetilde{\mathcal{J}}_{m} b^{1-v}\left(1+2 m_{3}^{*}\right)}{\Gamma(\beta)} \sup _{0 \leq \tau \leq s} \widehat{\mu}(\mathcal{K}(\tau)) \quad \text { (from Step 4 of Theorem 3.1), } \\
& \mathbb{I}_{2}=\frac{2 M M_{b} \widetilde{\mathcal{J}}_{m} b^{1-v}}{\Gamma(\beta)} \int_{0}^{t}(t-s)^{\beta-1} \widehat{\mu}\left(\left\{u_{g}(s)\right\}_{m=1}^{\infty}\right) d s \\
& \leq \frac{2 M M_{b} \widetilde{\mathcal{J}}_{m} b^{1-v}}{\Gamma(\beta)} \int_{0}^{t}(t-s)^{\beta-1} m_{4}(s) \frac{2 M \widetilde{\mathcal{J}}_{m}}{\Gamma(\beta)} \int_{0}^{b}(b-s)^{\beta-1} \widehat{\mu}\left(F_{m}(s)\right) d s \\
& \leq \frac{4 M^{2} \widetilde{\mathcal{J}}_{m}^{2} M_{b} M_{2} M_{4} b^{1-v}}{\Gamma(\beta)^{2}}\left(1+2 m_{3}^{*}\right) \sup _{0 \leq \tau \leq s} \widehat{\mu}(\mathcal{K}(\tau)), \\
& \mathbb{I}_{1}+\mathbb{I}_{2} \leq \frac{2 M \widetilde{\mathcal{J}}_{m} M_{2} b^{1-v}\left(1+2 m_{3}^{*}\right)}{\Gamma(\beta)}\left[1+\frac{2 M M_{b} M_{4} \widetilde{\mathcal{J}}_{m}}{\Gamma(\beta)}\right] \widehat{\mu}(\mathcal{K}(\tau)) .
\end{aligned}
$$

That is, $\widehat{\mu}(\widetilde{\Upsilon} \mathcal{K}(t)) \leq \frac{2 M \widetilde{\mathcal{J}}_{m} M_{2} b^{1-v}\left(1+2 m_{3}^{*}\right)}{\Gamma(\beta)}\left[1+\frac{2 M M_{b} M_{4} \widetilde{\mathcal{J}}_{m}}{\Gamma(\beta)}\right] \widehat{\mu}(\mathcal{K}(\tau))$. Therefore, using Mönch's condition, we get $\widehat{\mu}(\mathcal{K}) \leq \widehat{\mu}\left(\operatorname{conv}(\{0\} \cup(\widetilde{\Upsilon}(\mathcal{K})))=\widehat{\mu}(\widetilde{\Upsilon}(\mathcal{K})) \leq P^{*} \widehat{\mu}(\mathcal{K})\right.$, where $P^{*}$ is defined in (4.2), and hence Lemma 2.13 shows that (1.1)-(1.2) has a fixed point $\mathcal{K}$ in $F_{\ell}$. Hence, $z=g+\hat{\eta}$ is the mild solution of (1.1)-(1.2) satisfying $z(b)=z^{1}$. Consequently (1.3)-(1.4) is controllable on $\mathbb{N}$.

\section{Nonlocal conditions}

The nonlocal Cauchy problem for differential equation was first studied by Byszewski [69]. Their research is driven by imaginative enthusiasm and the manner in which these types of problems usually occur when proving practical applications. For example, material science and life sciences can be depicted by techniques for the differential framework subject to nonlocal limit conditions, the readers can refer to $[48,60,62,69,70]$. We presently expect that the nonlocal Sobolev-type Hilfer fractional integro-differential equations with control 
are as follows:

$$
\begin{aligned}
& D_{0^{+}}^{\alpha, \beta}[\mathcal{J} z(t)]=A z(t)+f\left(t, z_{t}, \int_{0}^{t} e\left(t, s, z_{s}\right) d s\right)+B u(t), \quad t \in \mathbb{N}=(0, b], \\
& \mathcal{I}_{0^{+}}^{(1-\alpha)(1-\beta)} z(0)=\phi+J\left(t_{i_{1}}, t_{i_{2}}, t_{i_{3}}, \ldots, t_{i_{k}}\right) \in \mathscr{B}_{l}, \quad 0<i_{k} \leq b, k=1,2, \ldots, n .
\end{aligned}
$$

The result is proved in relation to the following hypothesis:

$\left(\mathrm{A}_{4}\right)$ Function $J: \mathscr{B}^{n} \rightarrow \mathscr{B}$ is continuous, there exists $L_{i}(h)>0$ such that

$$
\left\|J\left(v_{1}, v_{2}, \ldots, v_{k}\right)-J\left(w_{1}, w_{2}, \ldots, w_{k}\right)\right\| \leq \sum_{k=1}^{n} L_{k}(J)\left\|v_{k}-w_{k}\right\|_{\mathcal{B}_{l}}
$$

for all $v_{k}, w_{k} \in \mathscr{B}_{l}$, and consider $L_{k}=\sup \left\{\|\|_{J}\left(v_{1}, v_{2}, \ldots, v_{k}\right) \|: v_{k} \in \mathscr{B}_{l}\right\}$.

Definition 5.1 A function $z:(-\infty, b] \rightarrow Z$ is a mild solution of (5.1)-(5.2) only if $z_{0}=$ $\phi+J\left(t_{x_{1}}, t_{x_{2}}, \ldots, t_{x_{n}}\right) \in \mathcal{B}_{l}$ on $(-\infty, 0]$ and

$$
\begin{aligned}
z(t)= & \mathcal{J}^{-1} \mathcal{S}_{\alpha, \beta}(t) \mathcal{J}\left[\phi+J\left(t_{x_{1}}, t_{x_{2}}, t_{x_{3}}, \ldots, t_{x_{n}}\right)(0)\right] \\
& +\int_{0}^{t}(t-s)^{\beta-1} \mathcal{J}^{-1} Q_{\beta}(t-s) f\left(s, z_{s}, \int_{0}^{s} e\left(s, \tau, z_{\tau}\right) d \tau\right) d s \\
& +\int_{0}^{t}(t-s)^{\beta-1} \mathcal{J}^{-1} Q_{\beta}(t-s) B u_{z}(s) d s, \quad t \in \mathbb{N},
\end{aligned}
$$

is satisfied.

Theorem 5.2 Assume that $\left(A_{0}\right)-\left(A_{4}\right)$ are satisfied, then (5.1)-(5.2) is controllable on $(0, b]$ if

$$
\frac{2 M \widetilde{\mathcal{J}}_{m} M_{2} b^{1-v}\left(1+2 m_{3}^{*}\right)}{\Gamma(\beta)}\left\{1+\frac{2 M M_{b} M_{4} \widetilde{\mathcal{J}}_{m}}{\Gamma(\beta)}\right\} \widehat{\mu}(\mathcal{K}(\tau))<1 \quad \text { for some } \frac{1}{2}<\beta<1
$$

is satisfied.

\section{Example}

Assume that the fractional evolution system with control is as follows:

$$
\left\{\begin{aligned}
& D_{0^{+}}^{\alpha, \frac{2}{3}}\left.z(\phi, \varsigma)-\frac{\partial^{2}}{\partial \varsigma^{2}} z(\phi, \varsigma)\right] \\
&=\frac{\partial^{2}}{\partial \varsigma^{2}} z(\phi, \varsigma)+U(\phi, \varsigma) \\
& \quad+g\left(\phi, \int_{-\infty}^{\phi} t_{1}(\sigma-\phi) z(\sigma, \varsigma) d \sigma, \int_{0}^{x} \int_{-\infty}^{0} \xi_{2}(r, \varsigma, \varepsilon-r) z(\varepsilon, \varsigma) d \varepsilon d r\right) \\
& \quad r \in[0, \pi], z \in(0, b], \\
&\left.\mathcal{I}^{(1-\alpha) \frac{1}{3}}[z(\phi, \varsigma)]\right|_{\varsigma=0}=z_{0}(\varsigma), \quad \varsigma \in[0, \pi] \\
& z(\phi, 0)=z(\phi, \pi)=0, \quad \phi \geq 0, \\
& z(0, \beta)=\phi(\beta), \quad 0 \leq \beta \leq \pi
\end{aligned}\right.
$$

where $D_{0^{+}}^{\alpha, \frac{2}{3}}$ denotes the Hilfer fractional derivative of order $\frac{2}{3}$, type $\alpha, g: \mathbb{N} \times[0,1] \times[0,1] \times$ $\mathbb{R} \rightarrow \mathbb{R}$ is continuous. 
To convert (6.1) into an abstract form, consider $Z=L^{2}(0, \pi)$ and $A: D(A) \subset Z \rightarrow Z$, let $\mathcal{J}: D(\mathcal{J}) \subset Z \rightarrow Z$ be defined as $A v=v^{\prime \prime}$, and $\mathcal{J} v=v-A, D(A), D(\mathcal{J})$ are given by

$\left\{v \in Z: v, v^{\prime}\right.$ are absolutely continuous, $\left.v(0)=v(\pi)=0\right\}$.

Additionally, $A$ and $P$ are presented as $A v=\sum_{m=1}^{\infty} n^{2}\left\langle v, z_{m}\right\rangle z_{m}, v \in D(A)$ and

$$
\mathcal{J} v=\sum_{m=1}^{\infty}\left(1+m^{2}\right)\left\langle v, z_{m}\right\rangle z_{m}, \quad v \in D(\mathcal{J})
$$

where $z_{m}(t)=\sqrt{\frac{2}{\pi}} \sin (m t), m=1,2, \ldots$ Also, for $z \in Z$, we have

$$
P^{-1} z=\sum_{m=1}^{\infty} \frac{1}{\left(1+m^{2}\right)}\left\langle z, z_{m}\right\rangle z_{m}, \quad A \mathcal{J}^{-1} z=\sum_{m=1}^{\infty} \frac{m^{2}}{\left(1+m^{2}\right)}\left\langle z, z_{n}\right\rangle z_{n}
$$

and $Q_{\beta}(x) z=\sum_{m=1}^{\infty} \exp \left(\frac{m^{2} t}{1+m^{2}}\right)\left\langle z, z_{n}\right\rangle z_{n}$.

Now, from [62] $z(t) z(s)=z(t+s)$ for $z \in Z, \widehat{\mu}(T(t) D) \leq \widehat{\mu}(D)$, where $T(t)$ is not compact and $\widehat{\mu}$ is the Hausdorff measure. Moreover, $\phi \rightarrow v\left(\phi^{\frac{2}{3}}+\sigma\right)$ is equicontinuous for $\phi \geq 0$. Define $f:[0, \pi] \times Z \rightarrow Z$ by

$$
\begin{aligned}
& e(\varkappa, \phi)=\int_{-\infty}^{\varkappa} \xi_{2}(\varkappa, r, \varepsilon) \phi(\varepsilon) d \varepsilon, \\
& f\left(\varkappa, \phi, \int_{0}^{\varkappa} e(\varepsilon, \phi) d \varepsilon\right)=\zeta\left(\phi, \int_{-\infty}^{\phi} \zeta_{1}(\sigma-\phi) u(\sigma, \gamma) d \sigma, \int_{0}^{\varkappa} e(\varepsilon, \phi)(r) d \varepsilon\right),
\end{aligned}
$$

and $D_{0^{+}}^{\alpha, \frac{2}{3}}(u)(\phi)(\beta)=\frac{\partial^{\frac{2}{3}}}{\partial \beta^{\frac{2}{3}}} u(\phi, \beta), u(\phi)(\varsigma)=u(\phi, \varsigma)$.

Let $B: U \rightarrow Z$ be defined by $(B w)(\phi)(\varsigma)=\mathcal{U}(\phi, \varsigma), 0<\varsigma<1$. For $\varsigma \in(0, \pi), W$ is given by

$$
W w(\varsigma)=\int_{0}^{1}(1-\phi)^{\frac{-1}{3}} P^{-1} Q_{\gamma}(1-\phi) \cup w(\phi, \varsigma) d \phi
$$

where

$$
Q_{\frac{2}{3}}=\frac{2}{3} \int_{0}^{\infty} \varsigma \Upsilon_{\frac{2}{3}}(\varsigma) w\left(x^{\frac{2}{3}} \varsigma\right) d \varsigma
$$

and for $\Upsilon \in(0, \infty)$,

$$
\begin{aligned}
& \Upsilon_{\frac{2}{3}}(\varsigma)=\frac{3}{2} \varsigma^{-1-\frac{-5}{2}} \bar{w}_{\frac{2}{3}}\left(\varsigma^{\frac{-3}{2}}\right), \\
& \bar{w}_{\frac{2}{3}}(\varsigma)=\left(\frac{1}{\pi}\right) \sum_{r=1}^{\infty}(-1)^{r-1} x^{-\frac{2}{3} r-1}\left[\frac{\Gamma\left(\frac{2}{3} r+1\right)}{r !}\right] \sin \left(\frac{2 r \pi}{3}\right) .
\end{aligned}
$$

Here, $\Upsilon_{\frac{2}{3}}$ is defined on $(0, \infty)$, that is,

$$
\Upsilon_{\frac{2}{3}}(\varsigma) \geq 0, \quad \varsigma \in(0, \infty) \text { as well as } \quad \int_{0}^{\infty} \Upsilon_{\frac{2}{3}}(\varsigma) d \varsigma=1,
$$

$f$ and $\mathcal{U}$ fulfills $\left(\mathrm{A}_{1}\right)-\left(\mathrm{A}_{3}\right)$. We conclude that (6.1) is controllable on $\mathbb{N}$. 


\section{Conclusion}

In this article, we have fundamentally focused on a class of Sobolev-type Hilfer fractional integro-differential framework with infinite delay, which generalized the RiemannLiouville fractional derivative. At first, we dealt with the new existence result of a mild solution with the assumptions that the framework satisfies the initial condition and noncompactness measure condition. Later, we have presented the controllability results of the thought about the fractional framework. In the end, we introduced an example to show the procured hypothetical results. We will try to investigate the neutral differential equation and controllability of a similar problem in our future research work.

\section{Acknowledgements}

The fourth and fifth authors would like to thank Azarbaijan Shahid Madani University. The authors would like to thank dear reviewers for their constructive comments which improved final version of this work.

\section{Funding}

Not applicable.

Availability of data and materials

Data sharing not applicable to this article as no datasets were generated or analyzed during the current study.

\section{Declarations}

Ethics approval and consent to participate

Not applicable.

\section{Consent for publication \\ Not applicable.}

Competing interests

The authors declare that they have no competing interests.

\section{Authors' contributions}

The authors declare that the study was realized in collaboration with equal responsibility. All authors read and approved the final manuscript.

\section{Author details}

${ }^{1}$ Department of Mathematics, School of Advanced Sciences, Vellore Institute of Technology, 632014 Vellore, Tamil Nadu, India. ${ }^{2}$ Department of Mathematics, College of Arts and Sciences, Prince Sattam bin Abdulaziz University, Wadi Aldawaser 11991, Saudi Arabia. ${ }^{3}$ Department of Applied Science, Rajkiya Engineering College Kannauj, Kannauj 209732, India. ${ }^{4}$ Department of Mathematics, Azarbaijan Shahid Madani University, Tabriz, Iran. ${ }^{5}$ Department of Medical Research, China Medical University Hospital, China Medical University, Taichung, Taiwan.

\section{Publisher's Note}

Springer Nature remains neutral with regard to jurisdictional claims in published maps and institutional affiliations.

Received: 16 June 2021 Accepted: 28 September 2021 Published online: 19 October 2021

\section{References}

1. Baleanu, D., Etemad, S., Rezapour, S.: On a fractional hybrid integro-differential equation with mixed hybrid integral boundary value conditions by using three operators. Alex. Eng. J. 59(5), 3019-3027 (2020). https://doi.org/10.1016/j.aej.2020.04.053

2. Thabet, S.T.M., Etemad, S., Rezapour, S.: On a coupled Caputo conformable system of pantograph problems. Turk. J. Math. 45(1), 496-519 (2021). https://doi.org/10.3906/mat-2010-70

3. Baleanu, D., Jajarmi, A., Mohammadi, H., Rezapour, S.: A new study on the mathematical modelling of human liver with Caputo-Fabrizio fractional derivative. Chaos Solitons Fractals 134, 109705 (2020). https://doi.org/10.1016/j.chaos.2020.109705

4. Adiguzel, R.S., Aksoy, U., Karapinar, E., Erhan, I.M.: Uniqueness of solution for higher-order nonlinear fractional differential equations with multi-point and integral boundary conditions. Rev. R. Acad. Cienc. Exactas Fís. Nat., Ser. A Mat. 115, 155 (2021). https://doi.org/10.1007/s13398-021-01095-3

5. Adiguzel, R.S., Aksoy, U., Karapinar, E., Erhan, I.M.: On the solution of a boundary value problem associated with a fractional differential equation. Math. Methods Appl. Sci. (2020). https://doi.org/10.1002/mma.6652

6. Adiguzel, R.S., Aksoy, U., Karapinar, E., Erhan, I.M.: On the solutions of fractional differential equations via Geraghty type hybrid contractions. Appl. Comput. Math. 20(2), 313-333 (2021) 
7. Alqahtani, B., Aydi, H., Karapinar, E., Rakocevic, V.: A solution for Volterra fractional integral equations by hybrid contractions. Mathematics 7(8), 694 (2019). https://doi.org/10.3390/math7080694

8. Baleanu, D., Rezapour, S., Saberpour, Z:: On fractional integro-differential inclusions via the extended fractional Caputo-Fabrizio derivation. Bound. Value Probl. 2019, 79 (2019). https://doi.org/10.1186/s13661-019-1194-0

9. Baleanu, D., Etemad, S., Rezapour, S.: A hybrid Caputo fractional modeling for thermostat with hybrid boundary value conditions. Bound. Value Probl. 2020, 64 (2020). https://doi.org/10.1186/s13661-020-01361-0

10. Baleanu, D., Mousalou, A., Rezapour, S.: On the existence of solutions for some infinite coefficient-symmetric Caputo-Fabrizio fractional integro-differential equations. Bound. Value Probl. 2017, 145 (2017). https://doi.org/10.1186/s13661-017-0867-9

11. Samei, M.E., Rezapour, S.: On a system of fractional q-differential inclusions via sum of two multi-term functions on a time scale. Bound. Value Probl. 2020, 135 (2020). https://doi.org/10.1186/s13661-020-01433-1

12. Thabet, S.T.M., Etemad, S., Rezapour, S.: On a new structure of the pantograph inclusion problem in the Caputo conformable setting. Bound. Value Probl. 2020, 171 (2020). https://doi.org/10.1186/s13661-020-01468-4

13. Rezapour, S., Samei, M.E.: On the existence of solutions for a multi-singular pointwise defined fractional q-integro-differential equation. Bound. Value Probl. 2020, 38 (2020). https://doi.org/10.1186/s13661-020-01342-3

14. Tuan, N.H., Mohammadi, H., Rezapour, S.: A mathematical model for COVID-19 transmission by using the Caputo fractional derivative. Chaos Solitons Fractals 140, 110107 (2020). https://doi.org/10.1016/j.chaos.2020.110107

15. Aydogan, S.M., Baleanu, D., Mousalou, A., Rezapour, S.: On high order fractional integro-differential equations including the Caputo-Fabrizio derivative. Bound. Value Probl. 2018, 90 (2018). https://doi.org/10.1186/s13661-018-1008-9

16. Kilbas, A.A., Srivastava, H.M., Trujillo, J.J.: Theory and Applications of Fractional Differential Equations. Elsevier, Amsterdam (2006)

17. Lakshmikantham, V., Leela, S., Vasundhara, D.J.: Theory of Fractional Dynamic Systems. Cambridge Academic Publishers, London (2009)

18. Deimling, K:: Multi-Valued Differential Equations. de Gruyter, Berlin (1992)

19. Pazy, A.: Semigroups of Linear Operators and Applications to Partial Differential Equations. Springer, New York (1983)

20. Podlubny, I.: Fractional Differential Equations. Academic Press, San Diego (1999)

21. Baleanu, D., Mohammadi, H., Rezapour, S.: Analysis of the model of HIV-1 infection of CD4+ T-cell with a new approach of fractional derivative. Adv. Differ. Equ. 2020, 71 (2020). https://doi.org/10.1186/s13662-020-02544-w

22. Sabetghadam, F., Masiha, H.P., Altun, I.: Fixed-point theorems for integral-type contractions on partial metric spaces. Ukr. Math. J. 68, 940-949 (2016). https://doi.org/10.1007/s11253-016-1267-5

23. Sabetghadam, F., Masiha, H.P.: Fixed-point results for multi-valued operators in quasi-ordered metric spaces. Appl. Math. Lett. 25(11), 1856-1861 (2012). https://doi.org/10.1016/j.aml.2012.02.046

24. Dineshkumar, C., Udhayakumar, R., Vijayakumar, V., Nisar, K.S.: A discussion on the approximate controllability of Hilfer fractional neutral stochastic integro-differential systems. Chaos Solitons Fractals 142, 110472 (2021). https://doi.org/10.1016/j.chaos.2020.110472

25. Mohan Raja, M., Vijayakumar, V., Udhayakumar, R.: Results on the existence and controllability of fractional integro-differential system of order $1<r<2$ via measure of noncompactness. Chaos Solitons Fractals 139, 110299 (2020). https://doi.org/10.1016/j.chaos.2020.110299

26. Mohan Raja, M., Vijayakumar, V., Udhayakumar, R., Zhou, Y.: A new approach on the approximate controllability of fractional differential evolution equations of order $1<r<2$ in Hilbert spaces. Chaos Solitons Fractals 141, 110310 (2020). https://doi.org/10.1016/j.chaos.2020.110310

27. Rezapour, S., Azzaoui, B., Tellab, B., Etemad, S., Masiha, H.P.: An analysis on the positivesSolutions for a fractional configuration of the Caputo multiterm semilinear differential equation. J. Funct. Spaces 2021, Article ID 6022941 (2021). https://doi.org/10.1155/2021/6022941

28. Mohan Raja, M., Vijayakumar, V., Udhayakumar, R.: A new approach on approximate controllability of fractional evolution inclusions of order $1<r<2$ with infinite delay. Chaos Solitons Fractals 141, 110343 (2020). https://doi.org/10.1016/j.chaos.2020.110343

29. Mahmudov, N.I., Udhayakumar, R., Vijayakumar, V.: On the approximate controllability of second-order evolution hemivariational inequalities. Results Math. 75, 160 (2020). https://doi.org/10.1007/s00025-020-01293-2

30. Shukla, A., Sukavanam, N., Pandey, D.N.: Approximate controllability of fractional semilinear control system of order $\alpha \in(1,2]$ in Hilbert spaces. Nonlinear Stud. 22(1), 131-138 (2015)

31. Shukla, A., Sukavanam, N., Pandey, D.N.: Approximate controllability of semilinear fractional control systems of order $\alpha \in(1,2]$ with infinite delay. Mediterr. J. Math. 13, 2539-2550 (2016). https://doi.org/10.1007/s00009-015-0638-8

32. Shukla, A., Sukavanam, N., Pandey, D.N.: Approximate controllability of fractional semilinear stochastic system of order $\alpha \in(1,2]$. J. Dyn. Control Syst. 23, 679-691 (2017). https://doi.org/10.1007/s10883-016-9350-7

33. Shukla, A., Sukavanam, N., Pandey, D.N.: Approximate controllability of semilinear fractional stochastic control system. Asian-Eur. J. Math. 11(6), 1850088 (2018). https://doi.org/10.1142/S1793557118500882

34. Singh, A., Shukla, A., Vijayakumar, V., Udhayakumar, R.: Asymptotic stability of fractional order $(1,2]$ stochastic delay differential equations in Banach spaces. Chaos Solitons Fractals 150, 111095 (2021). https://doi.org/10.1016/j.chaos.2021.111095

35. Vijayakumar, V., Udhayakumar, R., Kavitha, K.: On the approximate controllability of neutral integro-differential inclusions of Sobolev-type with infinite delay. Evol. Equ. Control Theory 10(2), 271-296 (2021). https://doi.org/10.3934/eect.2020066

36. Vijayakumar, V., Udhayakumar, R.: A new exploration on existence of Sobolev-type Hilfer fractional neutra integro-differential equations with infinite delay. Numer. Methods Partial Differ. Equ. 37, 750-766 (2021). https://doi.org/10.1002/num.22550

37. Williams, W.K., Vijayakumar, V., Udhayakumar, R., Nisar, K.S.: A new study on existence and uniqueness of nonlocal fractional delay differential systems of order $1<r<2$ in Banach spaces. Numer. Methods Partial Differ. Equ. 37(2), 949-961 (2021). https://doi.org/10.1002/num.22560

38. Hilfer, R:: Applications of Fractional Calculus in Physics. World Scientific, Singapore (2000)

39. Gu, H., Trujillo, J.: Existence of mild solution for evolution equation with Hilfer fractional derivative. Appl. Math. Comput. 257, 344-354 (2015). https://doi.org/10.1016/j.amc.2014.10.083 
40. Kavitha, K., Vijayakumar, V., Udhayakumar, R.: Results on controllability of Hilfer fractional neutral differential equations with infinite delay via measures of noncompactness. Chaos Solitons Fractals 139, 110035 (2020). https://doi.org/10.1016/j.chaos.2020.110035

41. Kavitha, K., Vijayakumar, V., Udhayakumar, R., Nisar, K.S.: Results on the existence of Hilfer fractional neutral evolution equations with infinite delay via measures of noncompactness. Math. Methods Appl. Sci. 44(2), 1438-1455 (2021). https://doi.org/10.1002/mma.6843

42. Kavitha, K., Vijayakumar, V., Udhayakumar, R., Sakthivel, N., Nisar, K.S.: A note on approximate controllability of the Hilfer fractional neutral differential inclusions with infinite delay. Math. Methods Appl. Sci. 44(6), 4428-4447 (2021). https://doi.org/10.1002/mma.7040

43. Kavitha, K., Vijayakumar, V., Shukla, A., Nisar, K.S., Udhayakumar, R.: Results on approximate controllability of Sobolev-type fractional neutral differential inclusions of Clarke subdifferential type. Chaos Solitons Fractals 151, 111264 (2021). https://doi.org/10.1016/j.chaos.2021.111264

44. Kavitha, K., Vijayakumar, V., Udhayakumar, R., Ravichandran, C.: Results on controllability of Hilfer fractional differential equations with infinite delay via measures of noncompactness. Asian J. Control (2021). https://doi.org/10.1002/asjc.2549

45. Subashini, R., Jothimani, K., Nisar, K.S., Ravichandran, C.: New results on nonlocal functional integro-differential equations via Hilfer fractional derivative. Alex. Eng. J. 59(5), 2891-2899 (2020). https://doi.org/10.1016/j.aej.2020.01.055

46. Vijayakumar, V., Henriquez, H.R.: Controllability results for a class of fractional semilinear integro-differential inclusions via resolvent operators. Numer. Funct. Anal. Optim. 39(6), 704-736 (2018). https://doi.org/10.1080/01630563.2017.1414060

47. Vijayakumar, V., Murugesu, R., Poongodi, R., Dhanalakshmi, S.: Controllability of second order impulsive nonlocal Cauchy problem via measure of noncompactness. Mediterr. J. Math. 14, 3 (2017). https://doi.org/10.1007/s00009-016-0813-6

48. Yang, M., Wang, Q.: Approximate controllability of Hilfer fractional differential inclusions with nonlocal conditions. Math. Methods Appl. Sci. 40(4), 1126-1138 (2017). https://doi.org/10.1002/mma.4040

49. Salim, A., Benchohra, M., Karapinar, E., Lazreg, J.E.: Existence and Ulam stability for impulsive generalized Hilfer-type fractional differential equations. Adv. Differ. Equ. 2020, 601 (2020). https://doi.org/10.1186/s13662-020-03063-4

50. Brill, H.: A semilinear Sobolev evolution equation in Banach space. J. Differ. Equ. 24(3), 412-425 (1997). https://doi.org/10.1016/0022-0396(77)90009-2

51. Chang, Y.K., Li, W.T.: Controllability of Sobolev type semilinear functional differential and integrodifferential inclusions with an unbounded delay. Georgian Math. J. 13(1), 11-24 (2006). https://doi.org/10.1515/GMJ.2006.11

52. Karapinar, E., Fulga, A., Rashid, M., Shahid, L., Aydi, H.: Large contractions on quasi-metric spaces with an application to nonlinear fractional differential equations. Mathematics 7(5), 444 (2019). https://doi.org/10.3390/math7050444

53. Lightbourne, J.H., Rankin, S.: A partial functional differential equation of Sobolev type. J. Math. Anal. Appl. 93(2), 328-337 (1983). https://doi.org/10.1016/0022-247X(83)90178-6

54. Mohammadi, H., Kumar, S., Rezapour, S., Etemad, S.: A theoretical study of the Caputo-Fabrizio fractional modeling for hearing loss due to mumps virus with optimal control. Chaos Solitons Fractals 144, 110668 (2021). https://doi.org/10.1016/j.chaos.2021.110668

55. Rezapour, S., Mohammadi, H., Jajarmi, A.: A new mathematical model for Zika virus transmission. Adv. Differ. Equ. 2020, 589 (2020). https://doi.org/10.1186/s13662-020-03044-7

56. Wang, J., Feckan, M., Zhou, Y.: Controllability of Sobolev type fractional evolution systems. Dyn. Partial Differ. Equ. 11(1), 71-87 (2014). https://doi.org/10.4310/DPDE.2014.v11.n1.a4

57. Abdelouaheb, A.: Asymptotic stability in Caputo-Hadamard fractional dynamic equations. Results Nonlinear Anal. 4(2), 77-86 (2021)

58. Lazreg, J.E., Abbas, S., Benchohra, M., Karapinar, E.: Impulsive Caputo-Fabrizio fractional differential equations in b-metric spaces. Open Math. 19(1), 363-372 (2021). https://doi.org/10.1515/math-2021-0040

59. Dineshkumar, C., Udhayakumar, R., Vijayakumar, V., Nisar, K.S., Shukla, A.: A note on the approximate controllability of Sobolev type fractional stochastic integro-differential delay inclusions with order $1<r<2$. Math. Comput. Simul. 190 1003-1026 (2021). https://doi.org/10.1016/j.matcom.2021.06.026

60. Ji, S., Li, G., Wang, M.: Controllability of impulsive differential systems with nonlocal conditions. Appl. Math. Comput. 217(16), 6981-6989 (2011). https://doi.org/10.1016/j.amc.2011.01.107

61. Nisar, K.S., Vijayakumar, V.: Results concerning to approximate controllability of non-densely defined Sobolev-type Hilfer fractional neutral delay differential system. Math. Methods Appl. Sci. (2021). https://doi.org/10.1002/mma.7647

62. Wang, J., Fan, Z., Zhou, Y.: Nonlocal controllability of semilinear dynamic systems with fractional derivative in Banach spaces. J. Optim. Theory Appl. 154, 292-302 (2012). https://doi.org/10.1007/s10957-012-9999-3

63. Benchora, M., Alssani, K., Nieto, J.: Controllability for impulsive fractional evolution inclusions with state-dependent delay. Adv. Theory Nonlinear Anal. Appl. 3(1), 18-34 (2019). https://doi.org/10.31197/atnaa.494662

64. Yan, B.: Boundary value problems on the half-line with impulses and infinite delay. J. Math. Anal. Appl. 259(1), 94-114 (2001). https://doi.org/10.1006/jmaa.2000.7392

65. Zhou, Y., Jiao, F.: Existence of mild solutions for fractional neutral evolution equations. Comput. Math. Appl. 59(3), 1063-1077 (2010). https://doi.org/10.1016/j.camwa.2009.06.026

66. Banas, J., Goebel, K.: Measure of Noncompactness in Banach Spaces. Dekker, New York (1980)

67. O'Regan, D., Precup, R.: Existence criteria for integral equations in Banach spaces. J. Inequal. Appl. 6(1), 77-97 (2001)

68. Monch, $\mathrm{H}$.: Boundary value problems for nonlinear ordinary differential equations of second order in Banach spaces. Nonlinear Anal., Theory Methods Appl. 4(5), 985-999 (1980). https://doi.org/10.1016/0362-546X(80)90010-3

69. Byszewski, L.: Theorems about the existence and uniqueness of solutions of a semilinear evolution nonlocal Cauchy problem. J. Math. Anal. Appl. 162(2), 494-505 (1991). https://doi.org/10.1016/0022-247X(91)90164-U

70. Byszewski, L., Akca, H.: On a mild solution of a semilinear functional-differential evolution nonlocal problem. J. Appl. Math. Stoch. Anal. 10(3), 265-271 (1997). https://doi.org/10.1155/S1048953397000336 